\title{
Desenvolvimento de Formulário para Registro do Processo de Cuidado Farmacêutico no contexto de um Hospital Materno-Infantil do Seridó Potiguar
}

\author{
Development of a Form to Register the Pharmaceutical Care Process in a \\ Maternal and Child Hospital in Seridó Potiguar
}

Recebido em: 20/03/2021 Aceito em: $14 / 05 / 2021$
Jefferson Shymenes Dantas CARDOSO; Almária Mariz BATISTA

Escola Multicampi de Ciências Médicas do Rio Grande do Norte.

Universidade Federal do Rio Grande do Norte (EMCM/UFRN).

Av. Dr. Carlindo Dantas, 540, $2^{\circ}$ andar, CEP 59300-000. Caicó, RN, Brasil.

E-mail:almariamariz@yahoo.com.br

\section{ABSTRACT}

Clinical indicators showed satisfactory results from the influence of the pharmacist's performance incorporated into the multi-professional team, contributing to the provision of responsible pharmacotherapy and patient-centered attention. However, this practice must be documented using pharmaceutical records in medical records for proper monitoring, prescription assessment and preparation of the pharmacotherapeutic profile, and guidance/recommendations to the healthcare team. A form was develped to register the Pharmaceutical Care process for the Maternity and Obstetrics Sector of a hospital in the Seridó Potiguar region. This descriptive and exploratory study is based on direct observation and consultation with Google Scholar, Pubmed, Scielo, Micromedex, and Uptodate databases for developing a registration form based on Drug-Related Problems and "Subject, Objective, Assessment, Planning" methodology. The form includes patient identification items, clinical and laboratory parameters, patient complaints, allergy records, medications used, drug-related problems, pharmaceutical assessment, and care plan. Development and use of this form, an instrument of health technology, aims to improve, despite the observed reality, the rational use of medicines, in addition to reinforcing the strategic position of the pharmacist as a health promoter, contributing to quality assistance to users of the Brazilian National Health System (Sistema Único de Saúde).

Keywords: pharmaceutical services; documentation; patient safety; hospital.

\section{RESUMO}

Indicadores clínicos demonstram resultados satisfatórios da influência de atuação do farmacêutico incorporado à equipe multiprofissional, contribuindo com a provisão da farmacoterapia responsável e atenção centrada no paciente. Esta prática, entretanto, deve ser documentada por meio de registro farmacêutico em prontuário para devido acompanhamento, avaliação da prescrição e elaboração do perfil farmacoterapêutico, além de orientações/recomendações à equipe assistencial de saúde. O objetivo deste 
estudo foi o desenvolvimento de um formulário para registro do processo de Cuidado Farmacêutico para o Setor de Maternidade e Obstetrícia de um hospital da região do Seridó Potiguar. Trata-se de estudo de caráter descritivo e exploratório, a partir de observação direta e consulta a bases de dados Google Scholar, Pubmed, Scielo, Micromedex e Uptodate para desenvolvimento de formulário de registro baseado em Problemas Relacionados a Medicamentos e método Subject, Objective, Assessment, Planning. O formulário inclui itens de identificação do paciente, parâmetros clínicos e laboratoriais, queixas do paciente, registro de alergia, medicamentos utilizados, problemas relacionados a medicamentos, avaliação farmacêutica e plano de cuidado. O desenvolvimento e a utilização deste formulário, um instrumento de tecnologia em saúde, visa a possibilidade de melhoria frente à realidade observada, ao uso racional de medicamentos, além de reforçar a posição estratégica do farmacêutico como promotor da saúde, contribuindo para assistência de qualidade aos usuários do Sistema Único de Saúde.

Palavras-chave: cuidados farmacêuticos; documentação; segurança do paciente; hospital.

\section{INTRODUÇÃO}

No Brasil, a saúde é garantida como direito fundamental de todo cidadão, consubstanciado na Lei $n^{\circ} 8080 / 90$, devendo o Estado prover condições indispensáveis a seu pleno exercício por meio da execução de políticas econômicas e sociais que visem a redução de riscos de doenças e estabelecimento de condições que assegurem acesso universal e igualitário a ações e serviços para sua promoção, proteção e recuperação, estando incluídas no campo de atuação do Sistema Único de Saúde (SUS), dentre outras, assistência terapêutica integral, inclusive farmacêutica (1).

Nesta perspectiva, é regulamentada a Política Nacional de Medicamentos (1998), a fim de garantir segurança, eficácia e qualidade dos produtos farmacêuticos, além de promover seu uso racional e acesso àqueles considerados essenciais (2).

Outrossim, a Política Nacional de Assistência Farmacêutica (2004) apresenta que Assistência Farmacêutica constitui um conjunto de ações voltadas a promoção, proteção e recuperação da saúde, tanto individual como coletiva, tendo o medicamento como insumo essencial e visando seu acesso e uso racional, na perspectiva de obtenção de resultados concretos e melhoria da qualidade de vida da população (3); entretanto, para muitos gestores o conceito de Assistência Farmacêutica permanece ainda centrado no binômio aquisição e distribuição de medicamentos $(2,3)$.
No contexto do uso racional de medicamentos, Hepler e Strand (1999) definiram Cuidado Farmacêutico como a provisão responsável do tratamento farmacológico com o propósito de alcançar resultados concretos que melhorem a qualidade de vida dos pacientes (4). E a Organização Mundial da Saúde (OMS) ampliou a perspectiva de cobertura do Cuidado Farmacêutico a toda comunidade e reconheceu o farmacêutico como um dispensador de atenção à saúde, que pode contribuir ativamente para prevenção de enfermidades e promoção da saúde em equipe multiprofissional. As discussões sobre este tema, na busca de entendimento do significado desta prática, continuam, e tem almejam adaptá-lo e integrá-lo aos modelos de saúde de cada país (5).

Neste sentido, cuidado farmacêutico é o modelo de prática que orienta a provisão de diferentes serviços farmacêuticos diretamente destinados ao paciente, à família e à comunidade, visando prevenção e resolução de problemas da farmacoterapia, uso racional e ótimo de medicamentos, promoção, proteção e recuperação da saúde, bem como prevenção de doenças e outros problemas de saúde (6).

A necessidade de integrar, o farmacêutico à equipe multidisciplinar para assistência integral à saúde vem sendo debatida desde a década 90, assim além das necessidades de comunicação objetiva e eficaz, surge também outras como a de padronizar os registros de suas atividades como intervenções e evolução do progresso realizadas no cuidado do paciente (7). 
Para tanto, o método Subject, Objective, Assessment, Planning (SOAP) é um modelo intervencionista não exclusivo para uso hospitalar, mas adaptado a esse, baseado no método Registo Médico Orientado por Problemas (RMOP), proposto por Lawrence Weed nos anos 60, em que as informações são registradas de forma livre, simples, sistematizada, possibilitando acompanhamento clínico, além de ser uma ferramenta de comunicação entre os membros da equipe de saúde, como forma de documentar a evolução do paciente $(8,9)$.

A prática do Cuidado Farmacêutico deve ser documentada pelo farmacêutico de forma clara, precisa, ordenada e completa as informações que o compete como resultado da assistência por ele prestada ao paciente, por meio de registro farmacêutico em prontuário após avaliação da prescrição, elaboração de perfil farmacoterapêutico do paciente e orientações à equipe assistencial de saúde. Neste registro constam problemas identificados, orientação farmacoterapêutica, sugestões de alteração de dose, dosagem, forma farmacêutica, técnica, via e horários de administração, dentre outros (10-12).

Ademais, a OMS, reconhecendo os potenciais riscos associados ao uso de medicamentos, lançou, em 2017, o Terceiro Desafio Global de Segurança do Paciente, Medicação Sem Danos, cuja meta foi reduzir em $50 \%$ os danos graves e evitáveis relacionados a medicamentos nos 5 anos seguintes, por meio do desenvolvimento de sistemas de saúde mais seguros e eficientes, com foco em 4 áreas, no caso, pacientes, medicamentos, profissionais de saúde, sistemas e práticas de medicação (13).

Assim, este estudo teve como objetivo o levantamento de informações para compilação e sistematização destas por meio de um formulário para registro do processo de Cuidado Farmacêutico destinado ao Setor de Maternidade e Obstetrícia de um hospital materno-infantil do Seridó Potiguar.

\section{MÉTODO}

Trata-se de um estudo de caráter descritivo e exploratório, desenvolvido entre fevereiro e outubro de 2020, em um hospital materno-infantil do município de Caicó, RN, Brasil, resultando na elaboração de um Formulário para Cuidado Farma- cêutico baseado no método $\operatorname{SOAP}(8,9)$ e no elenco de problemas relacionados a medicamentos (PRM) proposto pelo Consenso de Granada (14).

Para tanto, foi realizado um levantamento bibliográfico acerca do método SOAP nas bases de dados Google Scholar, Pubmed, Scielo, Micromedex e Uptodate associado à observação direta, in loco, do contexto do Hospital do Seridó, local de treinamento em serviço dos residentes do Programa de Residência Multiprofissional em Saúde Materno-Infantil, vinculado à Universidade Federal do Rio Grande do Norte (UFRN) e, assim, esta instituição de saúde foi definida como local de estudo.

O Hospital do Seridó é unidade de referência Materno-Infantil para 21 municípios da Região do Seridó, composta por 24 municípios (15). Situado na cidade de Caicó-RN, com total de 64 leitos distribuídos entre obstetrícia clínica (14), pediatria cirúrgica (2), pediatria clínica (12), clínica geral (18), cirúrgica geral (15) e unidade de isolamento (3).

Não há pactuação financeira com outros municípios para atendimento obstétrico, é uma unidade de atendimento exclusivo via SUS mantida pela pactuação entre União, Estado e gestão municipal de Caicó, RN (16).

O quadro de funcionários dispõe de 144 profissionais, dentre estes, 8 farmacêuticos, dos quais 5 institucionais distribuídos entre banco de leite (1), laboratório de análises clínicas (3) e farmácia central (1) e 3 residentes da Residência Multiprofissional em Saúde Materno-Infantil. O Setor de Obstetrícia foi eleito para destinação do formulário para Cuidado Farmacêutico (16).

Em se tratando de pesquisa sobre o aprofundamento teórico de situações que emergem espontânea e contingencialmente na prática profissional, não se fez necessária apreciação por Comitê de Ética e Pesquisa (17). Desta forma, os dados foram coletados mediante carta de anuência por parte da Direção do referido Hospital, sendo os dados registrados em diário de campo, que serviu de instrumento para posterior reflexão e análise das informações, considerando a literatura científica e o contexto da instituição em questão.

Feito isto, as referências foram levantadas, as informações foram compiladas e sistematizadas resultando na elaboração de um formulário para Cui- 
dado Farmacêutico, a ser disponibilizado ao Setor de Maternidade e Obstetrícia do referido hospital para registro das atividades clínicas desenvolvidas pelos farmacêuticos.

Finalizado o formulário para Cuidado Farmacêutico, foram propostas estratégias de sensibilização dos demais profissionais de saúde, gestores e usuários acerca da importância de identificar, prevenir e resolver PRM, bem como documentar estes problemas, a fim de alimentar o sistema de informações da instituição.

\section{RESULTADOS E DISCUSSÃO}

O SUS ainda enfrenta problemas importantes para assegurar o acesso e o uso racional de medicamentos à população. Algumas avaliações da assistência farmacêutica constataram que muitos problemas ainda precisam ser resolvidos. E apesar de algumas iniciativas terem tido sucesso, as dimensões do SUS impõem a necessidade de implantação de medidas mais estruturantes, com foco na promoção do uso racional de medicamentos (18-22).

A respeito da realidade local, foi constatada a falta de instrumento para organização e registro de informações acerca de intervenções farmacêuticas voltadas a identificação, prevenção e resolução de PRM, bem como de parâmetros de monitorização de resposta terapêutica e segurança da terapia farmacológica, provavelmente, em parte, devido à fragilidade da atuação clínica pelo farmacêutico, já que não há profissional designado para tal, contribuindo para possíveis prejuízos à efetividade e à segurança do tratamento medicamentoso na instituição.

A atuação clínica do farmacêutico nesta instituição praticamente foi consubstanciada pela Residência Multiprofissional em Saúde Materno-Infantil, por meio de farmacêuticos residentes que, entre outras atribuições diárias, realizam a prática clínica farmacêutica, entretanto, esta atuação ainda não se encontra totalmente amadurecida, principalmente, para os profissionais farmacêuticos do serviço.

Desta forma, depreende-se a possibilidade de consolidação desta prática por meio de um documento que instrumentalize e subsidie o registro sistemático de informações acerca do serviço clínico provido por farmacêutico, contribuindo para estruturação e padronização do processo de registro de condutas farmacêuticas em prontuário.

Farmacoterapia é um dos principais tipos de tratamento utilizados em hospitais. Para prática clínica, já existem ferramentas de caráter informatizado que possibilitam redução no tempo de atendimento, organização clara de informações, compartilhamento simultâneo de informações com a equipe multiprofissional e prevenção de erros, como o prontuário eletrônico do paciente $(23,24)$, mas, diante da realidade local, de prontuários manuais, a implantação de um formulário de registro da prática clínica do farmacêutico já caracteriza um avanço possível e necessário à melhoria da assistência na unidade hospitalar (Figura 1).

Este modelo requer a realização de anamnese farmacêutica, compreendida como procedimento de coleta de dados sobre o paciente, a fim de conhecer sua história de saúde, elaborar o perfil farmacoterapêutico e identificar possíveis PRM $(4,6,25)$. Desta forma, o farmacêutico obtém, inicialmente, informações do paciente, as quais são clinicamente interpretadas e confrontadas com dados do prontuário. Posteriormente, desenvolve-se um plano de cuidado, que será implementado e comunicado a toda equipe responsável pelo cuidado. Documentação, comunicação e colaboração com a equipe são pontos centrais deste processo (26).

Além disso, o método SOAP, acrônimo no qual cada letra representa uma das seções de registro da evolução diária, ou seja, dados subjetivos (S), dados objetivos (O), avaliação (A) e plano (P) $(9,27)$, constitui principal método de documentação utilizado em contextos hospitalar e ambulatorial, pois não necessita de formulário específico e o registro é simplificado (28).

Diante desta conjuntura, o modelo de Formulário proposto visa instrumentalizar a atuação clínica do farmacêutico, em busca da garantia de farmacoterapia efetiva, segura e conveniente, além de fornecer apoio à decisão clínica, viabilizando ao profissional de saúde melhores condições de atuação baseada em princípios científicos, melhor planejamento e avaliação da tomada de decisão, contribuindo para uniformidade e melhor qualidade do cuidado prestado $(23,29)$. 
Figura1. Proposta de formulário para registro de Cuidado Farmacêutico.

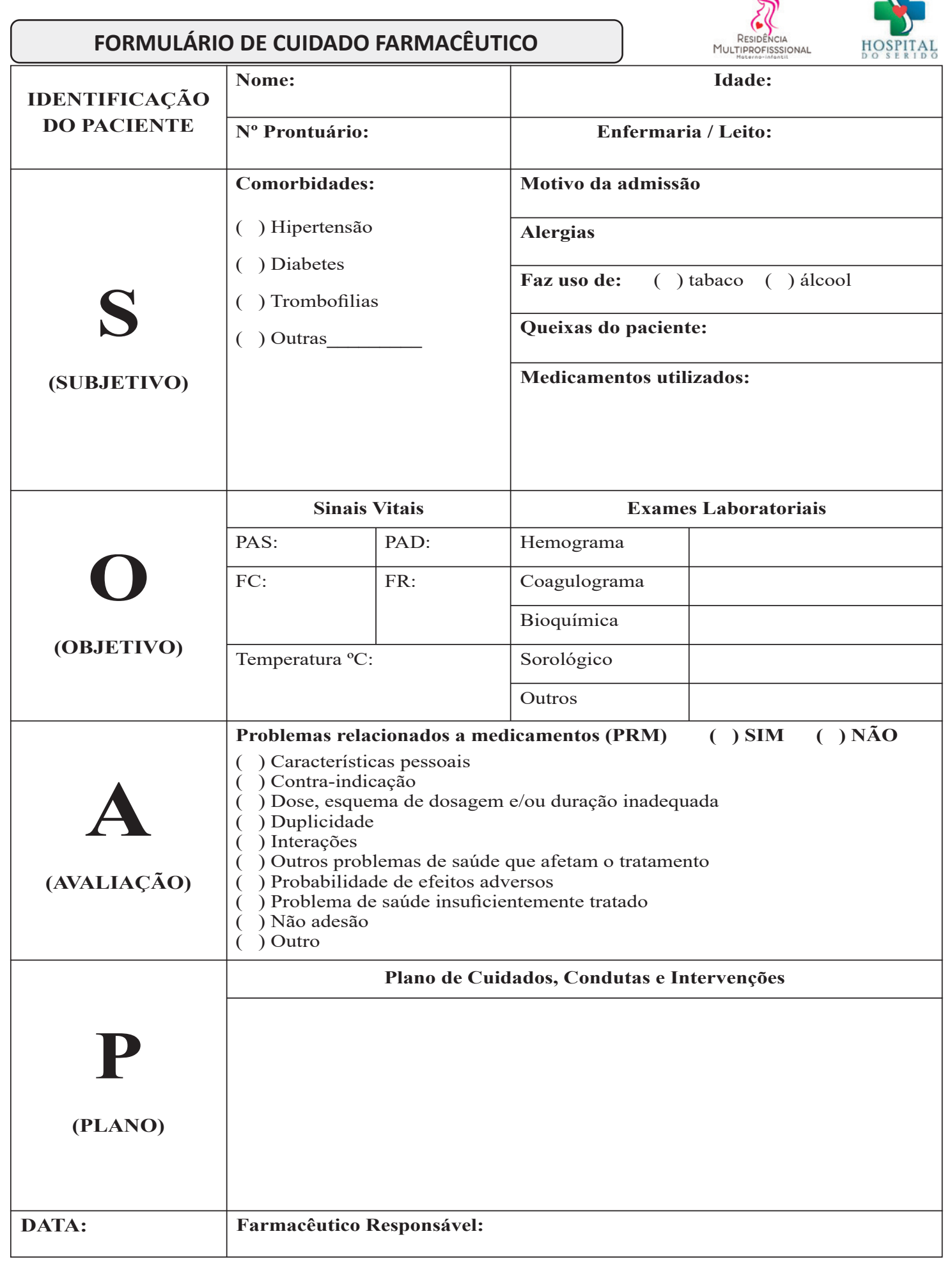

Esta proposta também vai ao encontro do preconizado pela Rede Cegonha, que visa organizar a assistência à saúde da mulher, o planejamento re- produtivo, a atenção humanizada a gravidez, parto e puerpério, além de ações voltadas à saúde da criança (30). Neste contexto, o registro de infor- 


\section{lutarma}

mações concernentes à terapia farmacológica por meio do Cuidado Farmacêutico vai tornar o processo mais humanizado.

O SOAP foi originalmente desenvolvido para subsídio à assistência médica, porém, ao longo do tempo muitos acrônimos surgiram a partir dele como Historical, Observation, Assessment, Planning (HOAP) (substitui parâmetros subjetivo e objetivo por histórico e observações); Subject, Objective, Assessment, Planning, Implementation, Evaluation, Revision (SOAPIER) (em Enfermagem, adiciona implementação, avaliação, revisão) e SOAP expandido (adiciona metas, monitoramento e educação). Para Farmácia, esta abordagem apresenta-se como referência para avaliação farmacêutica, focado na natureza de PRM, demonstrando importantes elementos de análise e possibilidade de registro da prática clínica farmacêutica (31).

Desta forma, inicia-se com Apresentação do Paciente. Em seguida, relacionam-se os Problemas de Saúde que o trouxeram à instituição, devendo ser colocados em ordem de importância clínica. Tratamento Medicamentoso rotineiro e atual seria o próximo item a ser listado para fins de análise de interações medicamentosas, duplicidade terapêutica, polifarmácia, alergia e dose (31). O próximo item relaciona-se ao Diagnóstico Farmacêutico, considerado processo cognitivo com foco na identificação de possíveis PRM, a partir de observações registradas anteriormente e evidências de evento indesejável (32). Seguem-se Resultados, que é a avaliação do alcance ou não dos objetivos propostos para o tratamento, Monitoramento, via testes laboratoriais, medidas clínicas e observações para feedback sobre evolução dos problemas de saúde e diagnóstico farmacêutico e Aconselhamento e Orientações ao Paciente para ajudá-lo a assumir com responsabilidade apropriada sua doença, além de fazê-lo reconhecer e lidar com possíveis efeitos adversos dos medicamentos (31).

Particularmente, Diagnóstico Farmacêutico requer mudança de paradigma, ou seja, reorientação de Diagnóstico baseado na identificação de PRM para prática que alie identificação de PRM e necessidades do paciente, o qual deve ser baseado em evidências clínicas e científicas. Para tanto, é essencial o acesso a informações Baseadas em Evidências (6).
No parâmetro subjetivo do SOAP são registradas informações do usuário/cuidador e histórico de prontuários. $\mathrm{Na}$ abordagem farmacêutica, são buscadas informações descritas sobre medicação do paciente, problemas de saúde relacionados, uso e adesão à terapia. No item Objetivo, estão dados objetivos mensuráveis, ou seja, informações clínicas observáveis como sinais vitais, resultados de exames laboratoriais e exames realizados pelos profissionais de habilitação para tal $(28,33)$.

A partir dos dados Subjetivos e Objetivos, no tópico Avaliação é esperada, do farmacêutico, a identificação de PRM, ou seja, eventos inerentes à farmacoterapia que, de fato ou potencialmente, possam interferir nos objetivos pretendidos. Por fim, o Plano de cuidados inclui alta, acompanhamento periódico, metas terapêuticas e pontos para resolução que envolvam PRM e parâmetros clínicos e laboratoriais que devem ser monitorados para necessidade, efetividade e segurança da farmacoterapia. Além disso, ações de educação em saúde para uso correto de medicamentos $(28,33)$.

O crucial é reconhecer dados essenciais às condições reais dos pacientes assistidos, para isto, além do documento e/ou do método, a expertise do profissional para preenchimento do documento também faz parte do processo, pois, à medida que são coletadas informações necessárias do paciente, organiza, documenta, analisa os dados, identifica problemas potenciais ou reais e os discute com a equipe multiprofissional, obter os resultados esperados torna-se mais propício (31).

As intervenções farmacêuticas em âmbito hospitalar apresentam desfechos positivos como em UTI obstétrica, na cidade de Teresina, PI em que, dentre 244 potenciais erros identificados, 42,6\% foram prevenidos, sendo os erros predominantes a omissão de dose ou medicamento $(23,8 \%)$, a sobredose $(16,4 \%)$, a diluição incorreta $(16,0 \%)$, a interação medicamentosa $(13,5 \%)$ e o medicamento não indicado/inadequado (5,7\%) (34).

Em um estudo desenvolvido em hospital de nível terciário, da cidade de São Paulo, SP, onde medicamentos, à exceção de prescrições de urgência, somente são dispensados após avaliação da prescrição médica pelo farmacêutico clínico, foram registradas 15.878 intervenções farmacêuticas/ 
ano (1.323/mês), predominando as relacionadas a ajuste de horário (48,31\%), ajuste de dose $(9,62 \%)$, ajuste de via de administração $(9,1 \%)$, duplicidade de prescrição médica $(8,79 \%)$ e medicamento em duplicidade (6.35\%). A aceitabilidade das intervenções pelos prescritores foi de 99,65\% (35).

Um estudo desenvolvido para analisar o perfil das intervenções realizadas pelo farmacêutico clínico em Hospital Universitário no Mato Grosso do Sul constatou-se que cuidados farmacêuticos podem contribuir para melhorar não só desfechos clínicos, mas também econômicos. Num universo de 737 prescrições analisadas, o percentual médio de aceitabilidade das intervenções farmacêuticas realizadas foi de $96,24 \%$, (36).

Considerando que documentação, comunicação e colaboração com a equipe são pontos centrais para efetividade do plano de cuidados, consequentemente, do cuidado farmacêutico (6), propõe-se como estratégias para efetividade da implantação do formulário sua apresentação a profissionais de saúde, gestores e usuários da instituição; treinamento dos farmacêuticos do serviço, a fim de capacitá-los acerca da aplicação do instrumento e matri- ciamento com a equipe multiprofissional, buscando a identificação de PRM, para posterior avaliação pelo farmacêutico.

\section{CONCLUSÃO}

A atuação clínica do farmacêutico contribui para o fortalecimento das atividades clínicas junto à equipe assistencial multiprofissional com atenção centrada no paciente. Neste sentido, a elaboração de um formulário para Cuidado Farmacêutico mostra-se importante diante do contexto local, marcado pela fragilidade da atuação clínica do farmacêutico, o que pode contribuir para possível aumento de riscos relacionados à farmacoterapia.

Portanto, faz-se importante também, a sensibilização de profissionais do serviço e gestores para que compreendam as dimensões dos impactos positivos inerentes ao Cuidado Farmacêutico frente à realidade, de forma a embasar um serviço que, aliado à equipe multiprofissional capacitada, envolvida e comprometida, realizem uma assistência de qualidade e humanitária.

\section{REFERÊNCIAS}

1. BRASIL. Lei no . 8080/90, de 19 de setembro de 1990. Dispõe sobre as condições para a promoção, proteção e recuperação da saúde, a organização e o funcionamento dos serviços correspondentes e dá outras providências. Diário Oficial da União, 20 de setembro de 1990. Seção 1. p. 18055 .

2. BRASIL. Ministério da Saúde. Portaria $n^{\circ} 3.916$, de 30 de outubro de 1998. Aprova a Política Nacional de Medicamentos. Diário Oficial da União, 10 de novembro de 1998. Seção 1.p. 18

3. BRASIL. Ministério da Saúde. Resolução no 338, 6 de maio de 2004. Aprova a Política Nacional de Assistência Farmacêutica. Diário Oficial da União, 20 de maio de 2004. Seção 1. p. 52.

4. Hepler CD, Strand LM. Oportunidades y responsabilidades en atención farmacêutica. Pharm Care Esp. 1999; $1(1): 35-47$.

5. OMS. El papel del farmacéutico en la atención a la salud: declaración de Tokio. Genebra: Organización Mundial de Salud. 1993.
6. CFF. Serviços farmacêuticos diretamente destinados ao paciente, à família e à comunidade: contextualização e arcabouço conceitual. Brasília: Conselho Federal de Farmácia. 2016.

7. Barnetts NMW, Hakim RC. Integration and assessment of the situation-background-assessment-recommendation framework into a pharmacotherapy skills laboratory for interprofessional communication and documentation. Curr Pharm Teach Learn. 2017;9(5):794-801. DOI: 10.1016/j.cptl.2017.05.023.

8. Weed LL. Medical records, patient care, and medical education. Ir J Med Sci. 1964;39(6):271-282. DOI: 10.1007/BF02945791.

9. Weed LL. Medical records that guide and teach. N Engl J Med. 1968;278(11):593-600. DOI: 10.1056/ NEJM196803142781105.

10. BRASIL. Resolução CFF $\mathrm{n}^{\circ} 555$, de 30 de novembro de 2011. Regulamenta o registro, a guarda e o manuseio de informações resultantes da prática da assistência farmacêutica nos serviços de saúde. Diário Oficial da União, 14 de dezembro de 2011. Seção 1. 
11. BRASIL. Resolução CFF nº 585, de 29 de agosto de 2013. Regulamenta as atribuições clínicas do farmacêutico e dá outras providências. Diário Oficial da União, 25 de setembro de 2013. Seção 1. p. 186.

12. BRASIL. Resolução $\mathrm{n}^{\circ} 572$, de 25 de abril de 2013. Dispõe sobre a regulamentação das especialidades farmacêuticas, por linhas de atuação. Diário Oficial da União, 06 de maio de 2013.

13. WHO. Medication without harm: WHO global patient safety challenge. Geneva: World Health Organization. 2017.

14. Comité de Consenso. Tercer Consenso de Granada sobre problemas relacionados con medicamentos (PRM) y resultados negativos associados a la medicación (RNM). Ars Pharm. 2007; 48(1):5-17.

15. IBGE. Instituto Brasileiro de Geografia e Estatística. Censo demográfico - 2010: organização dos territórios e organizações regionais. 2011.

16. CNES. Cadastro Nacional de Estabelecimentos de Saúde. [acessado em 29 de janeiro de 2021]. Disponível em: http://cnes.datasus.gov.br/pages/estabelecimentos/ ficha/hospitalar/2402002665778.

17. BRASIL. Resolução ${ }^{\circ} 510$, de 07 de abril de 2016. Dispõe sobre as normas aplicáveis a pesquisas em Ciências Humanas e Sociais cujos procedimentos metodológicos envolvam a utilização de dados diretamente obtidos com os participantes ou de informações identificáveis ou que possam acarretar riscos maiores do que os existentes na vida cotidiana, na forma definida nesta Resolução. Diário Oficial da União, no 98, 24 de maio de 2016. Seção 1. p. 44.

18. Lima-Dellamora EC, Caetano R, Osorio-de-Castro CGS. Dispensação de medicamentos do componente especializado em polos no Estado do Rio de Janeiro. Cienc Saude Colet. 2012;17(9):2387-2396. DOI: 10.1590/S1413-81232012000900019.

19. Guerin GD, Rossoni E, Bueno D. Itinerários terapêuticos de usuários de medicamentos de uma unidade de Estratégia Saúde da Família.Ciênc. Saúde Colet. 2012;17(11):30033010. DOI: 10.1590/S1413-81232012001100017.

20. Ungari AQ, Pereira LMV, Abramovicius AC, Pereira LRL, Forster AC, Grande MM. Estratégias para a garantia da integralidade na perspectiva do Componente Especializado da Assistência Farmacêutica. Rev Adm Saude. 2014;16(63):43-50. DOI: 10.5327/Z1519-167220 1400630002 .

21. Miai ET, Nogueira-Martins MCF. Farmacêuticos na atenção básica: estudo qualitativo sobre necessidades e possibilidades de qualificação dos profissionais para a integralidade do cuidado aos usuários-cidadãos. Bol Inst Saude. 2014;15(Supl):71-79.

22. Rover MRM, Vargas-Peláez CM, Farias MR, Leite SN. Da organização do sistema à fragmentação do cuidado: a percepção de usuários, médicos e farmacêuticos sobre o
Componente Especializado da Assistência Farmacêutica. Physis. 2016;26(2):691-711. DOI: 10.1590/S0103-73312 016000200017.

23. Crippa V, Dias DRC. Vantagens da implantação do prontuário eletrônico na segurança do paciente e na otimização do trabalho do farmacêutico hospitalar. Infarma Ciênc Farm. 2017;29(3):199-207. DOI: 10.14450/23189312.v29.e3.a2017.pp199-207.

24. Canêo PK, Rondina JM. Prontuário eletrônico do paciente: conhecendo as experiências de sua implantação. J. Health Inform. 2014;6(2):67-71.

25. Rivkin A. Thinking clinically from the beginning: early introduction of the pharmacists' patient care process. Am J Pharm Educ. 2016;80(10):164. DOI: 10.5688/ ajpe8010164.

26. JCPP. Joint Commission of Pharmacy Practitioners. Pharmacists' patient care process. 2014.

27. APA. Medication therapy management services: documenting patient care services. American Pharmacists Association. 2008.

28. Sando KR, Skoy E, Bradley C, Frenzel J, Kirwin, J, Urteaga E. Assessment of SOAP note evaluation tools in colleges and schools of pharmacy. Curr Pharm. 2017;9(4):576-584. DOI: 10.1016/j.cptl.2017.03.010.

29. Cipolle RJ, Strand LM., Morley PC. Pharmaceutical care practice: the patient centered to medication management. 3. ed. New York: McGraw-Hill, 2012.

30. BRASIL. Portaria $n^{\circ} 1.459$, de 24 de junho de 2011. Institui, no âmbito do Sistema Único de Saúde (SUS) - a Rede Cegonha. Diário Oficial da União.

31. Hurley SC. A method of documenting pharmaceutical care utilizing pharmaceutical diagnosis. Am J Pharm Educ. 1998;62(2):119-127.

32. Culbertson VL, Larson RA, Cady PS, Kale M, Force RW. A conceptual framework for defining pharmaceutical diagnosis. Am J Pharm Educ. 1997; 61:12-18.

33. Soares L, Farias MR, Leite SN, Campese M, Manzini F (Org.). Assistência farmacêutica no Brasil: política, gestão e clínica. v. 5. Florianópolis: Ed. da UFSC, 2016.

34. Souza, DMP. A contribuição da farmácia clínica nos indicadores clínicos e econômicos relacionados a farmacoterapia de mulheres internadas em uma unidade de terapia intensiva obstétrica. [Dissertação]. Teresina: Universidade Federal do Piauí. 2017.

35. Cardinal L, Fernandes C. Intervenção farmacêutica no processo da validação da prescrição médica. Rev. Bras. Farm. Hosp. Serv. Saúde. 2014;5(2):14-19.

36. Araujo EO, Viapiana M, Domingues EAM, Oliveira GS, Polisel CG. Intervenções farmacêuticas em uma Unidade de Terapia Intensiva de um Hospital Universitário. Rev. Bras. Farm. Hosp. Serv. Saúde. 2017;8(3):25-30. DOI: 10.30968/rbfhss.2017.083.005. 